\title{
Research about Renewable Materials in Indoor Decorate Engineering
}

\author{
Wei Liang ${ }^{1}$,Longxia Zhen ${ }^{2}$ \\ ${ }^{1}$ Environmental Art Design, Hebei Institute of Fine Arts, XinLe,ShiJiaZhuang,050700,China. \\ ${ }^{1}$ Environmental Art Design, Hebei Institute of Fine Arts,XinLe,ShiJiaZhuang,050700,China.
}

Xiaodong123@163.com

Keywords: Resources Save, Renewable materials.

\begin{abstract}
Modern society is highly productive society, as well as high consumption. How to limit resources constantly in the process of consumption, as far as possible to save resources, efficient used multiple times, maximum the value of resources? This is a worth further discussing issue for every field in modern society. Indoor decoration industry is widely regarded as a kind of high consumption of industry, popularize the use of renewable materials in the industry has a broader significance.
\end{abstract}

\section{Section One: the Definition of Renewable Materials}

Material is the material basis for human survival and development and it is used to make into useful components, components or material of the item, but not all substance can be referred to as material. Waste materials made from raw materials, used as initial materials for producing semi-finished products, components and finished products in the design of the such as plastic, metal, leather, stone, wood, etc. The definition about material diverse in different field. The material quote from this article refers to materials which used for interior decoration design .The so-called "renewable" indicate reprocessing the old items, keep the original characteristics itself to become the new product. Renewable materials refers to the remaining material after manufacturing process , or found, removed from the waste materials from scrap . These renewable materials are harmless and can be recycled. Renewable materials does not include the electrical energy, wind energy and so on a series of energy which is renewable materials.

Compared with the traditional interior materials, renewable materials has the following characteristics: (1) the environmental protection; The application of renewable material avoid the secondary development of materials, and the pollution to the environment. And has carried on the strict health disinfection to waste material, has the good environmental protection. (2) Sustainability; Renewable materials can be recycled used. (3) Economic; Renewable materials may be legacy from decoration field, also may be thrown into the garbage. (4) The unique aesthetic; Renewable materials perhaps can achieve special adornment effect which traditional materials unable to do.

How to recycle renewable materials? Whether it will be "junk" after being used? That the recycle value account. In the form of "recycling" ,items ware sold flow in the secondary market, it not only prolong the service life of the goods, but also can increase the way of products and services. Another recycled form is known as the "rebirth", the component which constitute the old goods rebirth via reproduce a new goods. Through the craft processing ,the intrinsic characteristics of the waste materials make the change of its shape to form a new product, which continues to play a waste material again use value.

Like the life traveler, we always think that waste won't impressed people, always in a hurry to come and then in a hurry to disappear from life. But surprisingly, when we are faced with problem about serious environmental pollution and resources shortage, it has become a very important "friend" of our side, is like your shadow, cannot leave, otherwise, we will lose the whole earth!

After old stuff recycling, on the premise of guarantee quality, can use the old itself or unloaded parts from the old for secondary use, and can reform the parts which can not use directly to become the new furniture. 
Industrial waste refers to all kinds of waste generated in industrial production, especially the construction waste. Today, with KunMing as an example, dismantle or reconstruct in the city will generate 38 million tons of construction waste. It is conceivable that city living space will be what appearance if the large amount of waste just throw away not handled in time, Therefore, we should actively the implementation of the construction waste in the utilization, it will reduce city waste pressure. There have report data that 1 tons of construction waste such as construction waste, such as debris, waste sand, concrete debris can be 0.45 tons of new wall material, the remaining $50 \%$ can be made into new road materials, $5 \%$ made of composite materials. At the same time, the garbage and other wastes should be classify in construction process, enterprises of renewable material recycling can prolong the life cycle of resources, avoid waste pollution maximum.

Since the industrial revolution, there generate big amount of waste by humans. According to data, about 3.1 billion tons of waste produced each year, of which 1.8 billion tons of garbage is non-toxic, 1.2 billion tons of civil garbage (living garbage and other debris), and 100 million tons of toxic waste. But life, construction, industrial waste recycling not only has economic value, and conducive to protect the environment. Nowadays, people have learned the extreme importance of material recycle, and waste recycling is becoming more and more popular, For artists, the use of waste is an inevitable choice, and they are also actively involved by the response, were held in the multiple renewable materials modification design contest both at home and abroad, and has found a creative way to convert the waste into human wealth. But still not mature, many design works in just waste the types of appearance design, unable to realize batch production.

At present, the domestic wastes are mixed garbage, all garbage waste is in a state of disorder. And the concept is similar like "dirty" ,"old" and "junk", therefore, in daily life, most people will ignore the old waste, get rid of it as "junk" directly, not to the consciousness of the recycling, it will affect the sustainable development of the human environment. In fact, we can see whether residential area or public places there are two trash cans and emissions in foreign countries, one is the recyclable bin, another is the unrecyclable bin. This method can categorizing waste. If we are to realize the importance of waste classification in recycling, perhaps we will never see garbage "a mountain" phenomenon.

"Waste" as useless waste in the world is not because it lost its function, but it left for its original site, temporarily abandoned. Puppet will find another new stage after leave the original occasions.

\section{Section Two: Classification of Renewable Materials}

With the development of interior decoration industry, renewable materials can be divided into: wood, cloth, paper, glass, plastic, metal.

2.1 waste wood: Wood is the most familiar to people, one of the most widely used building decoration materials, it performance a decisive role in interior design. Due to the color of the wood with a pleasant and pleasing to the eye, elegant and beautiful texture, good tactility, stronger and ease for processing, so the wood is the choice of furniture and interior design since the ancient times. As is known to all, China is a country Lack of forest resources, spending a lot of money on importing lumber each year. At the same time, we generate a large amount of wood waste everyday, such a large energy consumption of wood become the very serious problem. Therefore, reutilization of waste wood also gradually improve for designers.

There are various kinds of wood used in indoor decoration, bamboo as the main raw material for furniture and interior decoration. Wood used most widely in the field of material, it is suitable for any indoor space, wall board, ceiling, doors and windows, the floor, furniture. At the same time, it also can use in indoor furniture decoration, the inspiration of the series of works which furniture brand MADE is from the daily life, every piece of furniture is to use old waste wood collected from Manhattan area and purely handmade, they are simple and practical, reflecting the style of New York City. In addition, waste wood also can be made for welcome sign when entering into a buliding, also can be the cylinder of the bar and so on.

From the above example, you can see that wood is an ancient and eternal decorative material, it has the incomparable characteristics with other material, also has a powerful function. it has 
beautiful texture and color. Both reusing wood and new material can match each other. On interior decoration design, waste wood selection, new model design, construction technology to improve, etc, have put forward new challenges to designers.

2.2 the waste fabric :Decorative fabric is the main content of interior decoration design, it's also the important means for decorative environment space, to strengthen the atmosphere for interior. It give a taste about elegant, grace, simple and other different feelings by its unique texture, which form quiet harmonious atmosphere indoor. It provide comfortable living environment as well as bring people spiritual life about beauty.

Different decorative fabric can represent different decoration styles, and decorative fabric has a different purpose in interior design, mainly can be divided into: sofa cover, curtain, bedding, carpet, cushion, table cloth and so on. Different fabric, according to its purpose, function, and the use of different texture (such as cotton, hemp, wool, chemical fiber, silk and other materials), through the different technical means such as processing, cutting, forming different specifications, different colors and textures, so as to achieve the different artistic effects, meet people's different expectations.

In the interior environment, any kind of fabric can affect the overall space design, and is likely to change the indoor atmosphere, in addition to use the fabric flexibility and controllability, we should keep the consistency of the whole atmosphere space. Therefore, in the choose matching decorative fabric for interior design, the selected textiles which from pattern, texture, color and material will be around a certain subject or the author according to some kind of feelings, in the other words ,that's to create a coordinated feeling with the design theme.

According to the consistency principle when doing fabric decorative for interior space, the utilization rate for the rest of the decoration fabric is very high. When choose the same or similar fabric, the abandoned fabric can make another accessories collocation, both economical and beautiful. A chimney made of cloth. When light through the different pattern of cloth, often turning into different light shadow, that means, you can t define light and shadow in your willing. if the waste fabric does not match the design style and the surrounding environment, you can put it in the dark

With the concept of "light fixture, heavy decorate", decorative fabric is more and more popular in interior decoration design. It's a worthy pondering problem how to spend less cost under the condition of the noble, comfortable beautiful decoration space.

2.3 waste paper: Invention, production and use of paper for human has been thousands of years, those papers we used can wrap the earth several times enough, due to the production of paper burn trees is more difficult to count. The development of society and the prosperity of culture will stimulate further demand for paper, coupled with the constantly matured industrialized production technology, the production efficiency of paper improved, so that continue to reduce production cost, the price of the paper is in the change. the packaging of goods form is more and more various, such as food, cosmetics, clothing, footwear, packaging cartons and other packaging materials. There has class of daily paper, such as disposable paper cups, boxes, all kinds of advertising and so on.

Most people don't think paper is very solid, it may not make it big or strong items. Actually this is wrong. Once I have seen two student of architecture, with the construction principle, they made big paper house, after the experiment proved that it can withstand wind, stand, rain resistance had struck. Paper is something we need all the time with the improvement of our living standard and has huge consumption. But it is often ignored by the us and discarded easily, at present there still have 6 million tons of waste paper in our country has not been recycled, waste paper recovery rate is only $20 \%$ to $20 \%$, which is equivalent to waste of forest resources 1 million -3 million acres, and $1 \mathrm{t}$ recycling waste paper can regenerate $800 \mathrm{~kg}$ of new paper, can save 17 big tree. Whether such a large number astonish us?

\section{Section Three: Application of Renewable Materials in Interior Design}

3.1 application in household accessories design elements of renewable materials. With the continuous development of social economy, people pay more and more attention to improve their 
quality of life, consumption concept has also transformed from the original conservative to consumption type. Living space environment requirements gradually improve, to meet their spiritual requirements, and highlight the personal taste and social status. This concept is constantly development of interior design industry .At the same time it also stimulated the people's life, the pursuit of fashion. But also promotes the innovation of design.

Any kind of decorate all need to polish by accessories. Home Furnishing accessories design is to decorate after the completion of the fixture, to make combination design for handicrafts, textiles, lamp decoration, flower art and so on, a second display of indoor and arrangements, thus forming a new concept. We can say it is the detail design for household decoration design, through the meticulously arrangement about details indoor to foil out high taste of decoration and unique design. "Light fixture, heavy adornment" concept is advanced without doubt, the idea act the role of accessories design on the interior design stage, to make people focus more attention to accessories.

3.2 renewable materials used in the furniture.If indoor environment is an extension of the built environment, furniture is the ties connect interior space and human, in the progress of human civilization, people inseparable from the furniture. Furniture not only gives us convenient, but also gives us aesthetic feeling on the vision, can make us feel comfortable, warmth. Furniture design mainly around the "people-oriented" concept, present out the culture and mood. furniture in different interior space has different functions such as disjunctive space, create an atmosphere of space, adornment space and so on. Accord with the theme of the indoor environment, furniture meet people's spiritual needs and aesthetic requirements.

\section{Reference}

[1] The Rural Residential Technology Based on Renewable Energy System Design Research, Liu Wenhe, China Building Industry Press, 2014-5-1 1

[2] Evaluation Standard about Renewable energy Construction Application Engineering (GB/T50801-2013), National Standards of the People's Republic of China

[3] Normal Remote Monitoring System Renewable Energy and Construction , Lian Xiaoqin. China Building Industry Press

[4] Renewable Energy and Building Energy Utilization Technology, Hao Xiaoli, China Building Industry Ppress, 2014-7-1

[5] Renewable Eenergy and Building Energy Efficiency Technology, Electric Power Press, 2012-1-1 